\title{
For Hospital Readmissions, Hindsight is Not 20/20
}

\author{
Rosa R. Baier, MPH ${ }^{1,2}$ and Amal N. Trivedi, MD, MPH 2,3 \\ ${ }^{1}$ Center for Long-Term Care Quality \& Innovation, Brown University School of Public Health, Providence, RI, USA; '2Department of Health Services, \\ Policy \& Practice, Brown University School of Public Health, Providence, RI, USA; ${ }^{3}$ Providence VA Medical Center, Providence, RI, USA.
}

KEY WORDS: hospital; readmissions; rehospitalizations; communication; intervention.

J Gen Intern Med 31(11):1270-1

DOI: $10.1007 /$ s11606-016-3821-0

(C) Society of General Internal Medicine 2016

A s Deming and others have described, quality improvement is a continuous process that requires gathering information to define a problem and then testing and studying interventions that address its root causes. But how can providers intervene when they disagree about the contributing factors - or even the extent to which poor outcomes reflect suboptimal quality of care?

Transitional care is one area where there is broad debate about how to characterize problems and intervene. Because returning to the hospital following discharge is common, costly, and burdensome to patients and their caregivers, reducing readmission rates has emerged as an important quality goal. Many believe that some readmissions, perhaps $25 \%$ or more, ${ }^{1}$ could be prevented with better care: for example, if inpatient-outpatient communication occurred more seamlessly and if patients understood and acted on recommended followup. As a result, Medicare and other insurers are using readmission as a marker of poor quality care and financially penalizing hospitals (and soon nursing homes) for "higher than expected" 30-day readmission rates. This makes it increasingly pressing to identify effective strategies to prevent readmissions.

Recent evidence has documented that national readmission rates are, in fact, declining, ${ }^{2}$ though the precise mechanisms remain unclear. Although there are many well-known readmissions reduction programs targeting different factors, systematic reviews demonstrate programs' variable success and highlight the need for additional research to characterize the components of successful interventions. ${ }^{3,4}$

Implementing effective interventions to reduce readmissions is deceptively difficult. Consider, for instance, an action as seemingly simple as notifying primary care physicians (PCPs) that their patient was hospitalized. For these notifications to be effective, hospital staff must identify the correct $\mathrm{PCP}$ or practice location, ensure communications contain complete and accurate information and are successfully transmitted, and reach the right person - whether that person is

Published online August 3, 2016 the PCP, a nurse care manager, or someone else in the practice. The primary care practice must then act on the notification of the hospital visit, perhaps by providing pertinent information during the inpatient stay or by following up with the patient post-discharge.

Of course, preventing readmissions is far more complex than solely notifying the PCP. Numerous barriers affect patients' post-hospital care transitions and contribute to readmissions - far too many to detail. These include failure to prospectively identify and coordinate care for patients at highest risk for readmissions. They also include socioeconomic factors and lack of adequate access to care that affect patients' ability to obtain needed medications or services (medical or otherwise) and to keep follow-up appointments. Any single barrier can result from multiple root causes, each of which could be targeted for intervention. In light of this complexity, how can we implement systems change that improves communication and coordination for all patients, at all points in the care continuum? And how can we target or tailor assistance to those at highest risk?

Agreeing where to start is key. In this issue of the Journal of General Internal Medicine, Herzig et al. present results of a study that asked PCPs, admitting physicians, and discharging physicians to identify factors contributing to specific patients' readmissions and strategies to prevent readmissions. ${ }^{5}$ The resulting data highlight numerous opportunities for intervention, such as improved inpatient-outpatient communication and information transfer during and after the hospital stay. This could include not only notification of PCPs at hospital admission and provision of timely discharge summaries to PCPs, but also PCPs' reciprocal responsibility to provide inpatient physicians with information that informs inpatient care and discharge planning. Communication could occur via a phone call or conversation, though physicians' busy schedules may not always make that possible. Therefore, sharing of clinical records, ideally in real time, is essential. Without sharing of outpatient medical records, inpatient physicians may plan discharge without important details of a patient's clinical history, social context, and ability to self-manage care. Without timely transfer of discharge summaries, PCPs may be faced with caring for a recently discharged patient without knowing the reason for that patient's hospitalization, pending test results, and changes to the medication regimen. Such twoway communication is included in domains ${ }^{4}$ and best practices ${ }^{6}$ proposed by others. 
All three groups of physicians cited patient- (as opposed to physician- or system-) related factors with the greatest frequency, suggesting a need to identify interventions aimed at empowering and activating patients and caregivers during the chaotic time immediately following a hospital stay. The Care Transitions Intervention, which uses health coaches to teach patients to understand and navigate their conditions, medications, and follow-up, is one patient-focused intervention that has demonstrated both efficacy ${ }^{7}$ and effectiveness. ${ }^{8}$

While Herzig et al.'s results provide actionable information about contributing factors and strategies to prevent readmissions, the most striking finding is that agreement between physicians at the individual patient level was extremely low. The kappa statistic for concordance between any two physicians (PCP, admitting physician, or discharging physician) averaged only $0.13-0.16$. We do not know whose perceptions are correct, if anyone's, but the data underscore the difficulty of identifying the root causes of readmissions - even when considering a particular case in hindsight. PCPs, for example, chose factors related to the index hospital admission, continuity of care, provider communication, and social supports with greater frequency than either of their hospital counterparts. Such differences may not be surprising: as the authors note, inpatient and outpatient physicians have different vantage points and relationships with patients.

Of note, while the study surveyed physicians with multiple perspectives, it omitted one important voice: the patient's. Patients and caregivers can share valuable information about the factors contributing to their readmissions and the strategies they believe would be effective. ${ }^{9}$ What do patients believe are the key factors that contribute to their readmissions? Do their views agree with those of their providers? Stein et al. (2016) asked physicians and patients to identify the factors contributing to readmissions and also found poor agreement, ${ }^{10}$ suggesting that additional research is needed to incorporate patient perspectives into successful quality improvement efforts.

How can we improve readmissions without agreeing on the contributing factors? The study by Herzig et al. demonstrates that PCPs, admitting, and discharging physicians all have important, but diverse and divergent, perspectives about the reasons for readmissions. Enhancing communication among these providers and their patients is essential to improving care transitions and outcomes following a hospitalization. We need open dialogue to arrive at consensus about establishing expectations, prioritizing interventions, and obtaining buy-in for implementation.

Acknowledgements and Disclaimer: Dr. Trivedi was supported by the National Institute on Aging (grant nos. RO1 AG-044374 and PO1 AG-027296) and reports consulting for the Merck Manual. The views expressed in this article are those of the author and do not necessarily reflect the position or policy of the Department of Veterans Affairs or the US government.

Corresponding Author: Amal N. Trivedi, MD, MPH; Department of Health Services, Policy \& PracticeBrown University School of Public Health, 121 S. Main Street, Box G-S121-7, Providence, RI 02912, USA (e-mail: Amal_Trivedi@brown.edu).

\section{Compliance with Ethical Standards:}

Conflict of Interest: Dr. Trivedi reports receiving consulting fees to edit the Merck Manual.

\section{REFERENCES}

1. Auerbach AD, Kripalani S, Vasilevskis EE, et al. Preventability and causes of readmissions in a national cohort of general medicine patients. JAMA Intern Med. 2016;176(4):484-93. doi:10.1001/jamainternmed. 2015.7863.

2. Fingar K, Washington $\mathbf{R}$. Trends in hospital readmissions for four highvolume conditions, 2009-2013. Statistical Brief \# 196. Healthcare Cost and Utilization Project (HCUP) Statistical Briefs. Rockville, MD: Agency for Healthcare Research \& Quality; 2015.

3. Rennke S, Nguyen OK, Shoeb MH, Magan Y, Wachter RM, Ranji SR. Hospital-initiated transitional care interventions as a patient safety strategy: a systematic review. Ann Intern Med. 2013;158(5 Pt 2):433-40. doi:10.7326/0003-4819-158-5-201303051-00011.

4. Burke RE, Guo R, Prochazka AV, Misky GJ. Identifying keys to success in reducing readmissions using the ideal transitions in care framework. BMC Health Serv Res. 2014;14:423. doi:10.1186/1472-6963-14-423.

5. Herzig SJ, Schnipper JL, Doctoroff L, et al. Physician perspectives on factors contributing to readmissions and potential prevention strategies: A multicenter survey. J Gen Intern Med. 2016. doi:10.1007/s11606-0163764-5.

6. Baier R, Gardner R, Gravenstein S, Besdine R. Partnering to improve hospital-physician office communication through implementing care transitions best practices. Med Health RI. 2011;94(6):178-82.

7. Coleman EA, Parry C, Chalmers S, Min SJ. The care transitions intervention: results of a randomized controlled trial. Arch Intern Med. 2006;166(17): 1822-8.

8. Voss R, Gardner R, Baier R, Butterfield K, Lehrman S, Gravenstein S. The care transitions intervention: translating from efficacy to effectiveness. Arch Intern Med. 2011;171(14):1232-7. doi:10.1001/archinternmed.2011. 278.

9. Howard-Anderson J, Lonowski S, Vangala S, Tseng CH, Busuttil A, AfsarManesh N. Readmissions in the era of patient engagement. JAMA Intern Med. 2014;174(11):1870-2. doi:10.1001/jamainternmed.2014.4782.

10. Stein J, Ossman P, Viera A, Moore C, Brubaker BA, French J, Liles EA. Was this readmission preventable? Qualitative study of patient and provider perceptions of readmissions. South Med J. 2016;109(6):383-9. doi:10.14423/SMJ.0000000000000465. 